DOI: $10.15393 /$ j3.art.2015.2789

UDC 517.58, 517.51

F. QI, B. N. GuO

\title{
REMARKS ON COMPLETE MONOTONICITY OF A FUNCTION INVOLVING THE GAMMA FUNCTION
}

\begin{abstract}
In the note, the authors give several remarks on the paper in "Chen and Haigang Zhou On completely monotone of an arbitrary real parameter function involving the gamma function. Applied Mathematics and Computation, 2014, vol. 242, pp. 658663; DOI: 10.1016/j.amc.2014.05.034." By virtue of these, the authors point out several trivial extensions and generalizations and establish some new results on the complete monotonicity of a function involving the classical Euler gamma function.
\end{abstract}

Key words: remark, parameter, gamma function, completely monotonic function, logarithmically completely monotonic function, inequality

2010 Mathematical Subject Classification: 26A48, 33B15

Recall from [1, Chapter XIII], 2, Chapter 1], or [3, Chapter IV] that a function $f$ is said to be completely monotonic on an interval $I$ if it has derivatives of all orders on $I$ and satisfies

$$
0 \leq(-1)^{k-1} f^{(k-1)}(x)<\infty
$$

for $x \in I$ and $k \in \mathbb{N}$, where $f^{(0)}(x)$ means $f(x)$ and $\mathbb{N}$ stands for the set of all positive integers. The class of completely monotonic functions may be characterized by the famous Hausdorff-Bernstein-Widder theorem [3, p. 161, Theorem 12b]: A necessary and sufficient condition that $f(x)$ should be completely monotonic for $0<x<\infty$ is that

$$
f(x)=\int_{0}^{\infty} e^{-x t} \mathrm{~d} \alpha(t),
$$

where $\alpha(t)$ is non-decreasing and the above integral converges on $(0, \infty)$. 
Recall from [2, Chapter 5] and [4]-8] that an infinitely differentiable and positive function $f$ is said to be logarithmically completely monotonic on an interval $I$ if

$$
(-1)^{k}[\ln f(x)]^{(k)} \geq 0
$$

on $I$ for all $k \geq 1$.

It is well known [9, p. 31, (1.01)] that the classical Euler's gamma function may be defined by

$$
\Gamma(z)=\int_{0}^{\infty} t^{z-1} e^{-t} \mathrm{~d} t
$$

for $\operatorname{Re}(z)>0$. The logarithmic derivative of $\Gamma(z)$, denoted by

$$
\psi(z)=\frac{\Gamma^{\prime}(z)}{\Gamma(z)}
$$

is called the psi function or digamma function, and the derivatives $\psi^{\prime}(z)$ and $\psi^{\prime \prime}(z)$ are respectively called the trigamma and tetragamma functions. As a whole, the derivatives $\psi^{(m)}(z)$ for $m \in\{0\} \cup \mathbb{N}$ are called the polygamma functions. See [10, pp. 255-260].

In this note, we will give several remarks on the paper [11.

Remark 1. Let

$$
q(t)= \begin{cases}\frac{1}{t} \ln \Gamma(t+1), & t \neq 0 \\ -\gamma, & t=0\end{cases}
$$

on $(-1, \infty)$, where $\gamma=0.57721 \ldots$ denotes Euler-Mascheroni's constant. Comparing the function $f(t)$ defined by (3) below with

$$
h(t)=1-\ln (t+1)+q(t), \quad t>-1
$$

in $[11$, p. $659,(8)]$, whose complete monotonicity on $(-1, \infty)$ was established in [12], one may pose the following problem: What is the range of $\beta$ such that the function

$$
h_{\beta}(t)=1-\ln (t+\beta)+q(t)
$$

is completely monotonic, or equivalently, such that the function

$$
H_{\beta}(t)=\frac{1}{t+\beta} e^{q(t)}
$$

is logarithmically completely monotonic, on $(\max \{-1,-\beta\}, \infty)$ ? 
If the function $h_{\beta}(t)$ is completely monotonic on $(\max \{-1,-\beta\}, \infty)$, then its first derivative satisfies

$$
h_{\beta}^{\prime}(t)=\frac{e^{q(t)}\left[(\beta+t) q^{\prime}(t)-1\right]}{(\beta+t)^{2}} \leq 0
$$

which is equivalent to

$$
\beta \leq \frac{1}{q^{\prime}(t)}-t
$$

When $\beta>1$, the function $h_{\beta}(t)$ is defined on $(-1, \infty)$ and, by the L'Hôspital rule,

$$
\lim _{t \rightarrow-1^{+}}\left[\frac{1}{q^{\prime}(t)}-t\right]=\lim _{t \rightarrow-1^{+}}\left[\frac{t^{2}}{t \psi(t+1)-\ln \Gamma(t+1)}-t\right]=1 .
$$

This means that, when $\beta>1$, the function $h_{\beta}(t)$ is not completely monotonic on $(-1, \infty)$.

When $0<\beta \leq 1$, the function $h_{\beta}(t)$ is defined on $(-\beta, \infty) \supset(0, \infty)$ and

$$
h_{\beta}(t)=h(t)+\ln \frac{t+1}{t+\beta}
$$

for $t>-\beta$. When $\beta \leq 0$, the function $h_{\beta}(t)$ is defined on $(-\beta, \infty) \subseteq$ $\subseteq(0, \infty)$ and

$$
h_{\beta}(t)=f(t)+\ln \frac{t}{t+\beta}
$$

for $t>-\beta$. By virtue of

$$
\ln \frac{b}{a}=\int_{0}^{\infty} \frac{e^{-a u}-e^{-b u}}{u} \mathrm{~d} u
$$

in [10, p. 230, 5.1.32], it follows that the functions

$$
\ln \frac{t+1}{t+\beta}=\int_{0}^{\infty} \frac{e^{-(t+\beta) u}-e^{-(t+1) u}}{u} \mathrm{~d} u=\int_{0}^{\infty} \frac{e^{-\beta u}-e^{-u}}{u} e^{-t u} \mathrm{~d} u
$$

for $0<\beta \leq 1$ and

$$
\ln \frac{t}{t+\beta}=\int_{0}^{\infty} \frac{e^{-(t+\beta) u}-e^{-t u}}{u} \mathrm{~d} u=\int_{0}^{\infty} \frac{e^{-\beta u}-1}{u} e^{-t u} \mathrm{~d} u
$$


for $\beta \leq 0$ are both completely monotonic on $(-\beta, \infty)$. Since the sum of finitely many completely monotonic functions is still a completely monotonic function, the function $h_{\beta}(t)$ for $\beta \leq 1$ is completely monotonic on $(-\beta, \infty)$.

In conclusion, if and only if $\beta \leq 1$, the function $h_{\beta}(t)$ defined by (1) is completely monotonic, or equivalently, the function $H_{\beta}(t)$ defined by (2) is logarithmically completely monotonic, on the interval $(\max \{-1,-\beta\}, \infty)$.

Remark 2. The first main result of the paper [11] is [11, p. 659, Theorem 1] which reads that the function

$$
f_{\alpha}(x)=1-\ln (x+\alpha)+\frac{1}{x+\alpha} \ln \Gamma(x+\alpha+1)
$$

is completely monotone on $x>-\alpha$. Moreover, the function $f_{\alpha}(x)$ is decreasing on $x>-\alpha$, tends to 0 for $x \rightarrow \infty$ and to $\infty$ for $x \rightarrow-\alpha$.

Replacing $x+\alpha$ by $t$ we obtain that the function $f_{\alpha}(x)$ becomes

$$
f(t)=1-\ln t+\frac{1}{t} \ln \Gamma(t+1), \quad t>0,
$$

whose complete monotonicity has been proved in [6, p. 605, Theorem 2] which was cited in [11, p. 659, (5)]. Conversely, since $f(t)$ is completely monotonic on $(0, \infty)$, by definition, it is easy to see that $f(t+\alpha)=f_{\alpha}(x)$ is completely monotonic in $x \in(-\alpha, \infty)$.

Remark 3. The second main result of the paper [11] is [11, p. 659, Theorem 2] which states that the function

$$
g_{\alpha}(x)=\frac{[\Gamma(x+\alpha+1)]^{1 /(x+\alpha)}}{x+\alpha}
$$

is completely monotone on $x>-\alpha$.

Replacing $x+\alpha$ by $t$ we obtain that the function $g_{\alpha}(x)$ becomes

$$
g(t)=\frac{[\Gamma(t+1)]^{1 / t}}{t}, \quad t>0,
$$

whose logarithmically complete monotonicity has also been proved in [6, p. 605, Theorem 2] which was cited in [11, p. 659, (6)]. Conversely, since $g(t)$ is logarithmically completely monotonic on $(0, \infty)$, by definition, it is easy to see that $g(t+\alpha)=g_{\alpha}(x)$ is logarithmically completely monotonic 
in $x \in(-\alpha, \infty)$. Furthermore, the inclusion $\mathcal{L}[I] \subset \mathcal{C}[I]$ was proved and verified in [5, 6, 7, 13] once again, where $\mathcal{L}[I]$ and $\mathcal{C}[I]$ denote respectively the set of all logarithmically completely monotonic functions on an interval $I$ and the set of all completely monotonic functions on $I$. As a result, the complete monotonicity of $g(t)$ on $(0, \infty)$ and $g_{\alpha}(x)$ on $(-\alpha, \infty)$ are direct and simple consequences of the logarithmically complete monotonicity of $g(t)$ on $(0, \infty)$ and $g_{\alpha}(x)$ on $(-\alpha, \infty)$.

Remark 4. The function $f(t)$ defined by (3) above may be rearranged as

$$
f(t)=\ln \frac{e[\Gamma(t+1)]^{1 / t}}{t}=\ln [e g(t)], \quad t>0 .
$$

By definition, it is clear that the product between a positive scalar and a logarithmically completely monotonic function is still a logarithmically completely monotonic function and that a logarithm of a logarithmically completely monotonic function whose values are not less than 1 is surely a completely monotonic function. Consequently, the complete monotonicity of $f(t)$ may be derived from the logarithmically complete monotonicity of $g(t)$, it also may be conversely. In conclusion, the complete monotonicity of $f(t)$ and the logarithmically complete monotonicity of $g(t)$ are the same one.

Remark 5. In a word, for any function $f(x)$ on an interval $I$ and any real scalar $\alpha$, one should not regard the functions $f(x+\alpha)$ and $f(x)$ as different ones, and then one should not regard all the conclusions on the function $f(x+\alpha)$ as generalizations of those on $f(x)$.

Remark 6. This paper is a shortened and simplified version of the preprint [14].

Acknowledgment. The first author was partially supported by the National Natural Science Foundation of China under Grant No. 11361038. The authors appreciate the Editor-in-Chief, Professor Victor Starkov, and the Editorial Board for their kind and valuable comments on the original version of this paper.

\section{References}

[1] Mitrinović D. S., Pečarić J. E., and Fink A. M. Classical and New Inequalities in Analysis. Dordrecht; Boston; London: Kluwer Academic Publishers, 1993. DOI: $10.1007 / 978-94-017-1043-5$. 
[2] Schilling R. L., Song R., and Vondraček Z. Bernstein Functions-Theory and Applications. 2nd ed., de Gruyter Studies in Mathematics, 2012, vol. 37. DOI: $10.1515 / 9783110269338$.

[3] Widder D. V. The Laplace Transform. Princeton: Princeton University Press, 1946.

[4] Atanassov R. D. and Tsoukrovski U. V. Some properties of a class of logarithmically completely monotonic functions. C. R. Acad. Bulgare Sci., 1988, vol. 41, no. 2, pp. 21-23.

[5] Berg C. Integral representation of some functions related to the gamma function. Mediterr. J. Math., 2004, vol. 1, pp. 433-439. DOI: 10.1007/s00009-004-0022-6.

[6] Qi F. and Chen C. P. A complete monotonicity property of the gamma function. J. Math. Anal. Appl., 2004, vol. 296, pp. 603-607. DOI: 10.1016/j.jmaa.2004.04.026.

[7] Qi F. and Guo B. N. Complete monotonicities of functions involving the gamma and digamma functions. RGMIA Res. Rep. Coll., 2004, vol. 7, no. 1. Art. 8, pp. 63-72. URL: http: rgmia.org/v7n1.php.

[8] Qi F., Guo B. N., and Chen C. P. Some completely monotonic functions involving the gamma and polygamma functions. RGMIA Res. Rep. Coll., 2004, vol. 7, no. 1. Art. 5, pp. 31-36. URL: http://rgmia.org/v7n1.php.

[9] Olver F. W. J. Asymptotics and Special Functions. New York; San Francisco; London: Academic Press., 1974.

[10] Abramowitz M. and Stegun I. A. (Eds) Handbook of Mathematical Functions with Formulas, Graphs, and Mathematical Tables. Washington: National Bureau of Standards, Applied Mathematics Series, 1972, vol. 55, 10th printing.

[11] Chen B. and Zhou H.-G. On completely monotone of an arbitrary real parameter function involving the gamma function. Appl. Math. Comput., 2014, vol. 242, pp. 658-663. DOI: 10.1016/j.amc.2014.05.034.

[12] Vogt H. and Voigt J. A monotonicity property of the $\Gamma$-function. J. Inequal. Pure Appl. Math., 2002, vol. 3, no. 5. Art. 73. URL: http://www.emis.de/journals/JIPAM/article225.html.

[13] Guo B. N. and Qi F. A property of logarithmically absolutely monotonic functions and the logarithmically complete monotonicity of a powerexponential function. Politehn. Univ. Bucharest Sci. Bull. Ser. A Appl. Math. Phys., 2010, vol. 72, no. 2, pp. 21-30.

[14] Guo B. N. and Qi F. Remarks on complete monotonicity of a function involving the gamma function. ResearchGate Dataset, available online at URL: http://dx.doi.org/10.13140/RG.2.1.2378.2249. 
Received April 13, 2015.

In revised form, Jule 8, 2015.

Henan Polytechnic University

Jiaozuo City, Henan Province, 454010, China;

Inner Mongolia University for Nationalities

Tongliao City, Inner Mongolia Autonomous Region, 028043, China;

Tianjin Polytechnic University

Tianjin City, 300387, China

E-mail: qifeng618@gmail.com, qifeng618@hotmail.com

Henan Polytechnic University

Jiaozuo City, Henan Province, 454010, China

E-mail: bai.ni.guo@gmail.com, bai.ni.guo@hotmail.com 